\title{
Evaluation and Analysis for Maximum Lifespan of Wireless Sensor Networks by Energy-Efficient Design
}

\author{
Ms.Tejashri H. Mohite ${ }^{1}$ Prof.Dr.Noorullah Shariff ${ }^{2}$ \\ ${ }^{1}$ Assistant Professor in Electronics\& Telecommunication Engg. Dept,Dr.J.J.M.C.O.E.,Jaysingpur, \\ ${ }^{2} \mathrm{Head}$ of Dept. and Professor in Electronics and Communication EnggDept, SECAB Institute of Engineering \\ Technology,Bijapur,Karnataka
}

Abstract : Wireless Sensor Networks (WSNs) have used worldwide in the past few years and are now being used in health monitoring ,disaster management, defense, telecommunications, etc. Such networks are used in many industrial and consumer applications such as industrial process and environment monitoring, among others. A WSN network is a collection of specialized transducers known as sensor nodes with a communication link distributed randomly in any locations to monitor environmental parameters such as water level, and temperature. Each sensor node is equipped with a transducer, a signal processor, a power unit, and a transceiver. WSNs are now being widely used to monitor environmental parameters, including the amount of gas, water, temperature, humidity, oxygen level, dust, etc. The WSN for environment monitoring can be equivalently replaced by a multiple-input multiple-output (MIMO) relay network. Multi-hop relay networks have attracted significant research interest in recent years for their capability in increasing the coverage range. The network communication link from a source to a destination is implemented using the amplify-and-forward (AF) or decode-and-forward (DF) schemes. The AF relay receives information from the previous relay and simply amplifies the received signal and then forwards it to the next relay. On the other hand, the DF relay first decodes the received signal and then forwards it to the next relay in the second stage if it can perfectly decode the incoming signal. For analytical simplicity, in this thesis, we consider the AF relaying scheme and the results of this work can also be developed for the DF relay.

1.Introduction: A WSN is a collection of specialized transducers known as sensor nodes with a communication link distributed randomly in any locations to monitor parameters such as temperature, humidity, pressure, chemical concentration, pollutant levels, etc. Each sensor node is equipped with a transducer, a signal processor, a power unit, and a transceiver. The transducer converts the physical quantity into an electrical signal, and after processing the electrical signal, the transceiver transmits data to the other nodes. The power of each sensor node is supplied from an energy source, usually a battery, which defines the life-time of the overall network. The components of a WSN enable wireless connectivity and refer to a group of dedicated sensor nodes. A sensor node may vary in size from millimeter-size custom silicon to large-size integrated units. The range of wireless connectivity depends on the environment in which it is deployed, and it can be extended by adding relay nodes between a gateway and a leaf node for a particular topology [1]. The WSNs also have various resource constraints and challenges. Constraints include energy, bandwidth, memory, and processing capacity. Among them, energy consumption is of prime importance as each sensor node based on the number and type of the attached sensor components relies on the limited availability of battery power for data collection, processing, storage, transmission, and reception [22]. Moreover, energy consumption rate of each node depends on its distance from Base Station. The inequality of energy usage among the sensor nodes in the network affects the lifetime of the network for the intended applica-tion [23] [14]. Careful energy resource management is crucial for the WSNs deployed in remote areas for an extended period. Another specific challenge to WSN is the security attacks from the surrounding deployment area due to the broadcast nature of radio transmission. Due to the limited computing power of nodes, it is difficult to provide security and to protect the sensitive data from unauthorized access to WSN using public-key cryptography [15]. The climate and deployed environment also affect the efficiency in the WSN. This study aimed to develop a WSN system to monitor and collect the real-time hydrological and climatic data for the research study of mapping and modeling Variable Source Areas from a distantly located watershed. The specific objectives were to design and deploy a long-term, low-cost, and robust WSN system that can withstand harsh climatic conditions (extreme variation in temperature, high winds, rain, and snow) of humid and temperate climatic conditions

\section{Power optimization analysis:}

Energy Conserving Technique A WSN node has to perform three main tasks: sensing, data processing, and communication. Among them, communication consumes the major portion of the total energy, and it may also depend on the type of sensing. The recharging of the battery may be impossible in some cases because nodes are deployed 
usually in a hostile or remote location. So, the life-time of the network is directly associated with the energy consumption of the network nodes. In some cases, energy is added from the external sources by using solar cells but external sources may exhibit discontinuous behaviour which can affect the system performance. Energy consumption is taken into account by using efficient protocols during network activities based on the concept of switching off the components that are not needed in transmission [76]. A power management software is used to disconnect inactive components from the network when they are in sleeping mode. A node wakes up only when another node sends a network connection request; an alternative solution is that each node may remain active for a short time interval to accept connection requests from the djustant nodes. Collision avoidance schemes have been used recently to implement energy efficient transmission for Medium Access Control (MAC) protocols. MAC is an important technique that has been developed for wireless voice and data communication to enable the successful operation of the network. In the MAC protocol, the transmitter repeats the same message until it receive an acknowledgment (ACK) message from the receiver. The repetition of the same message consumes more energy in the network. The MAC protocol avoids collisions by allocating different time slot for each transmitter so that they can transmit at different time known as time division multiple access (TDMA) or it may assign different orthogonal codes to each source signals known as code division multiple access (CDMA).Alternatively, interference and additive Gaussian noise in the channel may corrupt the message, and the transmitter needs to resend the same signal until receiving an ACK confirmation from the receiver. In recent researches, MAC protocols are designed to reduce energy consumption by supporting scalability and collision avoidance.

\section{Sensors}

The pressure sensor used for the phase 3 WSN system shown in Figure 2(3) is a new series of sensor called the Freescale MPXV7007DP. The MPXV7007DP is a piezo-resistive monolithic silicon dual port pressure sensor. It has an output range of $(-2)$ to $2 \mathrm{kPa}$ with an accuracy of $\pm 2.5 \%$, with 0.5 to $4.5 \mathrm{~V}$ proportional output voltage. The operating temperature range for this sensor is $-40^{\circ} \mathrm{C}$ to $125^{\circ} \mathrm{C}$. The (E240-40761 10HS) $10 \mathrm{~cm}$ long (Decagon Devices, Inc.), high- frequency soil moisture sensor (Figure 2(4)) was selected for monitoring soil moisture. This capacitance type sensor has a large sphere of influence to measure the dielectric permittivity of the soil accurately. The Volumetric Water Content (VWC) measurement range of the $10 \mathrm{HS}$ sensor is $0 \%$ $57 \%$ for operating tem-perature between $0^{\circ} \mathrm{C}-50^{\circ} \mathrm{C}$ with an accuracy of $\pm 0.02 \mathrm{~m} 3 / \mathrm{m} 3( \pm 2 \%$ VWC) in any soil. The electric circuit inside the 10HS changes the capacitance measure-ment into a proportional millivolt $(\mathrm{mV})$ output. The high-frequency oscillator removes the soil type sensitivity of the sensor and thus, improves its ability to measure soil moisture in any soil.

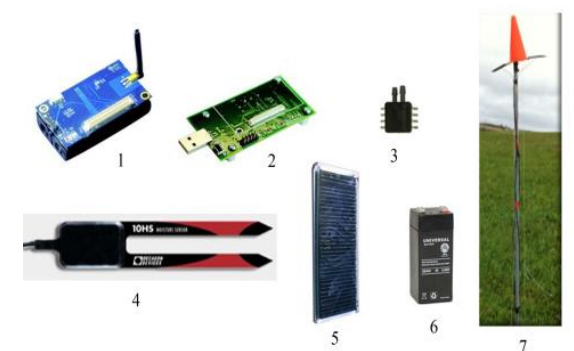

Figure 1 .IRIS Mote XM2110CA (1) gateway unit MIB510CA (2) pressure sensor. "Freescale" MPXV7007DP (3) soil moisture sensor (E240-40761) 10HS (4) 6V DC 100 $\mathrm{mA}$ solar panel (5) $4.0 \mathrm{~V}$ (4.5 Ah) lead-acid battery (6) assembly of a node in the field (7).

\section{Power Supply}

The third generation MICA2 nodes require a power range of 1.7 to $4.3 \mathrm{~V}$ DC supply for communication within its wireless network. After rigorous testing of various conventional and rechargeable batteries, 4.0 V (4.5 Ah) lead-acid batte-ries were found to be the most reliable for this application. These batteries lasted for about 30 days in the field under normal climatic conditions (Figure 2(6)). Solar panels of $14 \times 4 \times 0.5 \mathrm{~cm}$ with $6 \mathrm{~V} \mathrm{DC}$ open circuit voltage and a short circuit current output of $100 \mathrm{~mA}$ were used to recharge the batteries. These panels have two solder tabs with $7.5 \mathrm{~cm}$ long insulated leads to be connected to the batteries and weigh only 27 g. Each WSN node was provided with two solar pa-nels to charge the batteries and maintain the supply voltage within a specified range to extend the battery life and the WSN operation, as shown in Figure 2.

5. The Sturdiness of Node Assembly Each wireless node was housed in a sturdy and watertight PVC housing $(80 \times 50$ $\times 25 \mathrm{~mm}$ ) to withstand harsh temperatures, winds, and rain in the field. Mois-ture absorption packages were also placed within the casing to prevent humid conditions and to ensure that moisture does not collect on the electronics. The node housing was attached to a $3.0 \mathrm{~m}$ long and $25 \mathrm{~mm}$ diameter PVC pipe. This pipe was connected to a $450 \times 450 \times 100$ mm wooden pedestal.

The wooden pedestal was secured in the field using four 29 $\mathrm{cm}$ long PVC plugs. A glow sign cone was attached on top of the node to protect the PVC housing from rain, snow and for providing prominent visibility (Figure 2(7)). A pair of solar panels was attached to this cone. This modified node setup was found to be very sturdy and resistant to severe weather conditions. The overall node components, sensors, and node assembly in the field are shown in Figure 2(7). 2.5. 6.Communication Connectivity

The nodes were elevated $3.0 \mathrm{~m}$ above ground level to increase communication connectivity so that the crop height and the depressed areas did not interfere with the line of sight connectivity between the nodes. Increased height of the nodes improved connectivity between the nodes and resulted in a decreased number of required nodes and reduced the overall cost of the WSN system. The hardware components were purchased directly from the distributors, 
and data acquisition boards for the IRIS Mote were designed and fabricated in the labor-atory in order to increase the cost-effectiveness. The assembling of WSN com-ponents was carried out in the department workshop.

\section{MSP432 Optimizations}

With growing complexity in the microcontroller (MCU) applications, minimizing theoverall energy consumption of a system is one of the most challenging problems [23]. Multiple

aspects, such as the hardware components used onboard and the application software, must beconsidered [23]. Some obvious generic technique, such as reducing the frequency, might notsignificantly reduce the energy consumption independently but taken as a whole, the result mightbe significant, as there are many interdependencies across these components [23].

The MSP432microcontroller includes several power enhancements features to reduce the overall powerconsumption. The device provides various options and power configurations that enabledevelopers to optimize the power consumption for a specific application.

\section{Energy Measurement}

Debuggers with EnergyTrace technology support include a new and unique way of continuously measuring the energy supplied to a target microcontroller that differs considerably from the well-known method of amplifying and sampling the voltage drop over a shunt resistor at discrete times. A softwarecontrolled dc-dc converter is used to generate the target power supply. The time density of the dc-dc converter charge pulses equals the energy consumption of the target microcontroller.

A built-in on-the-fly calibration circuit defines the energy equivalent of a single dc-dc charge pulse. Figure 3 shows the energy measurement principle. Periods with a small number of charge pulses per time unit indicate low energy consumption and thus low current flow. Periods with a high number of charge pulses per time unit indicate high energy consumption and also a high current consumption. Each charge pulse leads to a rise of the output voltage VOUT, which results in an unavoidable voltage ripple common to all dc-dc converters.

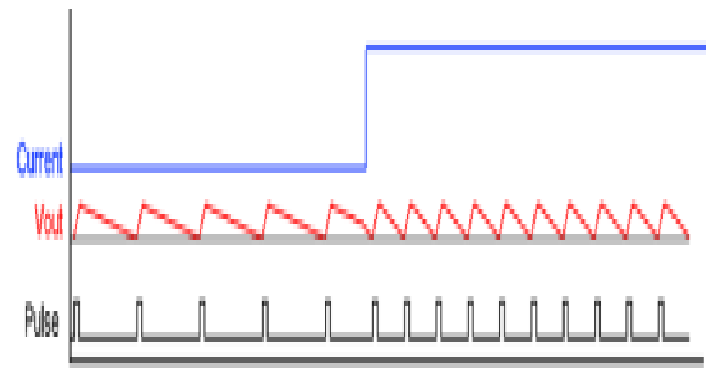

Figure 3. Pulse Density and Current Flow

Above figure shows the energy measurement principle. Periods with a small number of charge pulses per time unit indicate low energy consumption and thus low current flow.
Periods with a high number of charge pulses per time unit indicate high energy consumption and also a high current consumption. Each charge pulse leads to a rise of the output voltage VOUT, which results in an unavoidable voltage ripple common to all dc-dc converters. The benefit of sampling continuously is evident: even the shortest device activity that consumes energy contributes to the overall recorded energy. No shunt-based measurement system can achieve this.

\subsection{Reducing operating voltage}

Power is the product of current and voltage. By reducing the supply voltage in certain applications, power consumption can be reduced. The constraint here is that the minimum voltage requirement is met.

\subsection{Reducing the operating frequency}

Power and current consumption aredirectly proportional to the operating frequency. Inmost cases, higher operating frequency means the CPU can execute the codes and complete thetask faster. In a real-time scenario, many applications are time-dependent or event-driven. Thereare cases when CPU is running faster in an idle loop waiting for a certain event to trigger. TheCPU can spend a lot of time waiting for serial data to come in at a lower baud rate. This canconsume additional power which can be reduced by reducing the operating frequency.

\subsection{Maximizing the sleep time}

There are two modes generally considered in low power designs- active mode and low power mode. During active mode, it executes the designed tasks. The low power mode is the period where there is minimal activity, other than timekeeping or waiting for an interrupt or event to wake up to the active mode. Low power mode consumes less current compared to active mode. There are different low power modes in the MSP432 microcontroller where current consumption can be as low as $700 \mathrm{nA}$, while active mode current can be up to several milliamps.Maximizing the low power mode can significantly reduce the power consumption.

The objective of this work is to optimize the overall power consumption dissipated by the system. Different hardware and software optimization techniques were applied to reduce the overall power consumption. After the power optimization process, a buck-boost converter was selected to reduce power using a 2 Volt solar panel and a 3.7 Volt Liion rechargeable battery.

The LTC3106 IC was used to manage power of the system when there was enough sunlight, as well as in the absence of sunlight, and have the system run continuously without any interruption.

\section{Results and Calculations}

After the optimization process the minimum current required during transmission wasreduced from 105 $\mathrm{mA}$ to about $78 \mathrm{~mA}$. The system was set to sleep mode whenever it wasneeded. The power required to the system was properly managed. The LCD was included in the system for user convenience. The backlight of the LCD was turned 
off after it was on for 20 seconds so that the backlight of LCD does not drain unnecessary currents.

\section{Minimum current calculations-}

As discussed earlier, the minimum current required for transmission:

Without any optimization $=105 \mathrm{~mA}(1)$

After optimization $=78 \mathrm{~mA}(2)$

Current reduced $=\mathrm{I}$

$\mathrm{I}=$ InitialvalueFinalValue

\section{$* 100 \%$}

\section{Initialvalue}

$=105 \mathrm{~mA}-78 \mathrm{~mA}$

----------------------* $100 \%$

$105 m A$

$=25.71 \%(3)$

The minimum current requirement for running the system can be reduced by $25.71 \%$ bymanaging the individual components.

The pins in LTC3106 are connected in such a way that the solar panel was set as aprimary source and the rechargeable battery as a secondary source. The aim was to make surethat if the solar energy was enough to make the overall system run, the excess energy was usedto charge the battery. The system would run via battery in the absence of solar power. Two solar panels were connected in parallel to get the required amount of current for the load during thedaytime.

The ADC was used for measuring the voltage from the solar panel, LTC and the batteryand the current from the solar panel and the LTC. The single ended mode of the ADC was usedfor measuring the voltage. The voltage from the solar cells and the MSP432 was measured withthe MSP432 ADC but for the battery voltage, a voltage divider circuit and a buffer op-amp wereused to drop down the maximum voltage of the battery that could go as high as 4.2 Volts whenfully charged below 3.3 Volts. The MSP432 pins can measure a maximum of 3.3 Volts. A 10KOHM and a 36 KOHM resistors were used for the voltage divider circuit to drop down thevoltage. An LM324 op-amp was used as a buffer to match the impedance level of the battery andthe MSP432. The obtained value was converted to the equivalent battery voltage using a linear

regression equation.

The equation was generated with a known voltage source values and thenwas verified. For current measurement, $1 \mathrm{OHM}$ resistor was placed as shown in the figure belowand the differential voltage across each resistor was measured using the differential mode ofADC of the MSP432. The battery current was not measured directly but the current available tocharge the battery can be calculated using the solar current and LTC current. When there was nosolar energy available, the battery was sourcing the current to the LTC. When solar energy wasavailable there were some current left for charging the battery, after boosting the output voltage. The currents and voltages were displayed on the system LCD.

For this research, a rechargeable Li-ion battery was chosen as a secondary source to powerthe system. A 2000mAh Ni-MH battery was tested, but it was mostly available in 1.2 Volts, thusrequired multiple batteries connected in series for providing the required current. Whileconnecting the batteries in series the output voltage gets added. While testing $2 \mathrm{Ni}-\mathrm{MH}$ batteriesin series, it was only providing current for some time, but not enough time required for ESP8266during its setup process in the beginning. Even though supercapacitors provided the surge currentthe initial Wi-Fi connectivity time could last up to a minute, as a result, the system was not ableto startup all the time. Thus, Li-ion battery was a better option.

The currents drawn by different load resistors were observed when powered from a fullcharged 3.7 Volt, $350 \mathrm{mAh} \mathrm{Li-ion}$ battery serving as a secondary source to the LTC3106. Fromthe observation across different load resistor, it can be observed that the LTC3106 has an output current limitation of $180 \mathrm{~mA}$ when the LTC is powered from a Li-ion battery that when fullycharged has an output voltage of $4.2 \mathrm{~V}$

\section{Conclusion:}

A system was designed to collect- the temperature, pressure and humidity data sensor. Thedata was transmitted to a workstation in a remote location by connecting to a router with anInternet connection. The current consumption of the system was measured, and successive stepswere taken to optimize the overall system. The process started with optimization in the MSP432board, followed by BME sensor, LCD display and the Wi-Fi module. The sleep mode feature ofthe MSP432 was used to set the system to sleep when the system is not transmitting any data ordisplaying the values. The minimum current required during transmission was reduced by $25.71 \%$ after the optimization process.The LTC3106 was used for managing power to the system. The solar panel was used as a primary source and a Li-ion battery as a secondary source. The current and voltage coming fromthe solar panels, coming out form the LTC3106 and the voltage from the battery was measuredusing the ADC in the MSP432 board. When solar energy was available, the LTC was consuming some current to boost the voltage, some of the difference was available to charge the battery. Thebattery charging current should be in the opposite polarity from the current supplied by thebattery.Based on observations on different lighting condition a $350 \mathrm{mAh}$ battery was chosen fortesting the charging and discharging rate. The system transmitted the sensor readings to theGoogle Spreadsheets continuously for about 8 days (193 hours) on battery power alone with anaverage current consumption of $1.81 \mathrm{~mA}$ due to the low duty cycle of 0.0001388 of peak currentdemand. Then the system was tested in a controlled environment where the charge lost duringdark hours was compensated by the solar power received during day hours. 


\section{REFERENCES}

[1] G. Han, L. Liu, J. Jiang, L. Shu, G. Hancke, "Analysis of energyefficientconnected target coverage algorithms for industrial WirelessSensor Networks," IEEE Trans. on Industrial Informatics, vol. 13, no. 1,pp. 135-143, 2017.

[2] K. Sundareswaran, V. Vigneshkumar, P. Sankar, S.P. Simon, P.S.R.Nayak, S. Palani, "Development of an improved P\&O algorithm assistedthrough a colony of foraging ants for MPPT in PV system," IEEE Trans.on Industrial Informatics, vol. 12, no. 1, pp. 187-200, 2016.

[3] E. Koutroulis and K. Kalaitzakis, "Novel battery charging regulationsystem for photovoltaic applications," IEE Proceedings - Electric PowerApplications, vol. 151, no. 2, pp. 191-197, 2004.

[4] W. Xu, Y. Zhang, Q. Shi, X. Wang, "Energy management and crosslayer optimization for wireless sensor network powered byheterogeneous energy sources," IEEE Trans. on WirelessCommunications, vol. 14, no. 5, pp. 2814-2826, 2015.

[5] F. Mansourkiaie, L.S. Ismail, T.M. Elfouly, M.H. Ahmed, "Maximizinglifetime in wireless sensor network for structural health monitoring withand without energy harvesting," IEEE Access, vol. 5, pp. 2383-2395,2017.

[6] M. Magno, D. Boyle, D. Brunelli, E. Popovici, L. Benini, "Ensuringsurvivability of resource-intensive sensor networks through ultra-lowpower overlays," IEEE Trans. on Industrial Informatics, vol. 10, no. 2,pp. 946-956, 2014.

[7] J. Zhang, Z. Li, S. Tang, "Value of information aware opportunistic dutycycling in solar harvesting sensor networks," IEEE Trans. on IndustrialInformatics, vol. 12, no. 1, pp. 348-360, 2016.

[8] Y. Li, Z. Jia, S. Xie and F. Liu, "Dynamically reconfigurable hardwarewith a novel scheduling strategy in energyharvesting sensor networks,"IEEE Sensors Journal, vol. 13, no. 5, pp. 2032-2038, 2013.

[9] B. Zhang, R. Simon, H. Aydin, "Harvesting-aware energy management

for time-critical wireless sensor networks with joint voltage andmodulation scaling," IEEE Trans. on Industrial Informatics, vol. 9,no. 1, pp. 514-526, 2013.

[10] S.K.K. Ng, J. Zhong, J.W.M. Cheng, "Probabilistic optimal sizing ofstand-alone PV systems with modeling of variable solar radiation andload demand," in Power and Energy Society General Meeting, pp. 1-7,2012.

[11] C. Paravalos, E. Koutroulis, V. Samoladas, T. Kerekes, D. Sera, R.Teodorescu, "Optimal design of photovoltaic systems using high timeresolutionmeteorological data," IEEE Trans. on Industrial Informatics,vol. 10, no. 4, pp. 2270-2279, 2014.

[12] M. Alsayed, M. Cacciato, G. Scarcella, G. Scelba, "Multicriteria optimalsizing of Photovoltaic-Wind turbine grid connected systems," IEEETrans. on Energy Conversion, vol. 28, no. 2, pp. 370-379, 2013.

[13] B.J. Abdelhak, E. Najib, H. Abdelaziz, F. Hnaien, F. Yalaoui, "Optimumsizing of hybrid $\mathrm{PV} /$ wind/battery using fuzzyadaptive genetic algorithmin real and average battery service life," in International Symposium onPower Electronics, Electrical Drives, Automation and Motion

(SPEEDAM), pp. 871-876, 2014.

[14] O.H. Mohammed, Y. Amirat, M. Benbouzid, A.A. Elbaset, "Optimaldesign of a PV/fuel cell hybrid power system for the city of Brest inFrance," in 2014 International Conference on Green Energy,pp. 119-123, 2014.

[15] M. Mirjafari, R.S. Balog, R. Turan, "Multiobjective optimization of theDC-DC stage of a module-integrated inverter based on an efficiencyusage model," IEEE Journal of Photovoltaics, vol. 4, no. 3, pp. 906-914,2014.

[16] A.C. Nanakos, G.C. Christidis, E.C. Tatakis, "Weighted efficiencyoptimization of flybackmicroinverter under improved boundaryconduction mode (i-BCM)," IEEE Trans. on Power Electronics, vol. 30,no. 10, pp. 5548-5564, 2015.

[17] G. Adinolfi, G. Graditi, P. Siano, A. Piccolo, "Multiobjective optimaldesign of photovoltaic synchronous Boost converters assessingefficiency, reliability, and cost savings," IEEE Trans. on IndustrialInformatics, vol. 11, no. 5, pp. 1038 1048, 2015.

[18] M. Kasper, D. Bortis, J.W. Kolar, "Classification and comparativeevaluation of PV panel-integrated DC-DC converter concepts," IEEETrans. on Power Electronics, vol. 29 , no. 5, pp. 2511-2526, 2014.

[19] F. Beltrame, F. H. Dupont, H. C. Sartori, E. C. Cancian, C. Rech, J. R.Pinheiro, "Efficiency optimization of DC/DC boost converter applied tothe photovoltaic system," in 39th Annual Conference of the IEEEIndustrial Electronics Society (IECON), pp. 706-711, 2013.

[20] Y. Louvrier, P. Barrade, A. Rufer, "Weight and efficiency optimizationstrategy of an interleaved DC-DC converter for a solar aircraft," in $13^{\text {th }}$ European Conference on Power Electronics and Applications (EPE),pp. 1-10, 2009.

[21] I. Mandourarakis, E. Koutroulis, "Design optimization of a RES-basedpower-supply system for Wireless Sensor Networks", in 2015,15 International Conference on Environment and Electrical Engineering(EEEIC), pp. 1-6, 2015.

[22] Mahgoub, I. and Ilyas, M. (2016) Sensor Network Protocols. CRC Press, Taylor and Francis Group, Boca Raton.

[23] Akyildiz, I.F., Su, W., Sankarasubramaniam, Y. and Cayirci, E. (2002) Wireless Sensor Networks: A Survey. Computer Networks, 38, 393-422. https://doi.org/10.1016/S13891286(01)00302-4

[24] Bogena, H.R., Huisman, J.A., Oberdorster, C. and Vereecken, H. (2007) Evaluation of a Low-Cost Soil Water Content Sensor. Journal of Hydrology, 344, 32-42. https://doi.org/10.1016/j.jhydrol.2007.06.032

[25] Prabhu, S.R.B., Pradeep, M. and Gajendran, E. (2017) Monitoring Climatic Condi-tions Using Wireless Sensor Networks. A Multidisciplinary Journal of Scientific Research \& Education, 3, 179-184.

[26] Jing, W. and Tingting, L. (2015) Application of Wireless Sensor Network in Yangtze River Basin Water Environment Monitoring. Control and Decision Conference, 27, 59815985. https://doi.org/10.1109/CCDC.2015.7161882

[27] Khairnar, P.P., Gaikwad, D.V., Kale, S.R., Madane, M.T. and Giri, M.B. (2016) Wireless Sensor Network Application in Agriculture for Monitoring Agriculture Production Process. International Journal of Advanced Research in Computer Engineering \& Technology, 5, 2278-1323.

[28] Burrell, J., Brooke, T. and Beckwith, R. (2004) Vineyard Computing: Sensor Net-works in Agricultural Production. IEEE Pervasive Computing, 3, 38-45. https://doi.org/10.1109/MPRV.2004.1269130

[29] Verma, R. (2013) A Survey on Wireless Sensor Network Applications, Design In-fluencing Factors \& Types of Sensor Network. International Journal of Innovative Technology and Exploring Engineering, 3, 2278-3075.

[30] Barrenetxea, G., Ingelrest, F., Schaefer, G., Vetterli, M., Couach, O. and Parlange, M. (2008) Sensor Scope: Out-ofthe-Box Environmental Monitoring. IEEE Com-puter Society, 332-343. https://doi.org/10.1109/IPSN.2008.28 